\title{
14. APPENDICES
}

\section{APPENDIX 1}

\section{DESCRIPTION OF SELECTED BIRTH DEFECTS}

The following include descriptions of some of the birth defects included in this report :

Anencephaly

Spina bifida

Encephalocele

Hydrocephalus

Buphthalmos

Hypospadias

Epispadias

Chordee

Talipes equinovarus

Polydactyly

Syndactyly

Craniosynostosis

Exomphalos

Gastroschisis

Cystic hygroma
Absence of the cranial vault, with the brain tissue completely missing or markedly reduced.

Defective closure of the bony encasement of the spinal cord, through which the spinal cord may protrude.

Protrusion of brain through a congenital opening in the skull

Dilatation of the cerebral ventricles accompanied by an accumulation of cerebral fluid within the skull.

Enlargement and distension of the fibrous coats of the eye.

The opening of the urethra lies on the underside of the penis or on the perineum.

Absence of the upper wall of the urethra. The opening of the urethra lies on the dorsum of the penis in males, and anterior to or onto the clitoris in females.

Downward bowing of the penis.

A deformity of the foot in which the heel is elevated and turned outward.

Presence of additional fingers or toes on hands or feet.

Attachment of adjacent fingers or toes on hands or feet.

Premature closure of the sutures of the skull.

Herniation of the abdominal contents into the umbilical cord.

A defect in the abdominal wall not involving the umbilicus and through which the abdominal contents herniate.

A sac, cyst or bursa distended with fluid.

\section{APPENDIX 2}

\section{BIRTH DEFECT EXCLUSION LIST}

The following is a general list of minor defects and non-structural disorders which are excluded from the NSW Birth Defects Register:

\section{Abnormal palmar creases}

Accessory nipples

Balanced chromosomal translocation (unless occurring with structural defects)

Birthmarks (single, $<4 \mathrm{cms}$ diameter)

Bronchopulmonary dysplasia

Cerebral palsy

Clicky hips

Congenital infections (unless occurring with structural defects)

Congenital neoplasms/tumours (exception: cystic hygroma)

Developmental disability

Deviated nasal septum

Fetal alcohol syndrome

Glucose-6-phosphate dehydrogenase (G6PD) deficiency

Haemophilia

Heart murmurs (functional)

Hernia (epigastric, hiatus, inguinal, umbilical)

Hydrocele (testis)

Hypoplastic lung (less than 37 weeks gestation)

Imperforate hymen

Inborn errors of metabolism other than phenylketonuria, galactosemia and congential hypothyroidism.

Intrauterine growth retardation

Low birth weight

Meconium ileus

Minor ear anomalies

Minor finger/hand anomalies

Minor toe/foot anomalies

Muscular dystrophies \& myopathies

Oesophageal reflux

Patent ductus arteriosus (less than 37 weeks gestation)

Pilonidal sinus

Sacral dimples

Single umbilical artery (unless occurring with structural defects)

Skin tag

Strabismus

Talipes (exception: those requiring surgery)

Tongue tie

Undescended testes (exception: those requiring surgery)

Webbing of 2nd \& 3rd toes

Wide sutures 


\section{APPENDIX 3}

MATERNAL COUNTRIES OF BIRTH AND COUNTRY OF BIRTH GROUPS

\section{English speaking}

Australia

Christmas Island

Cocos (Keeling) Islands

Norfolk Island

New Zealand

United Kingdon

Channel Islands

Isle of Man

Ireland

Bermuda

Canada

United States of America

South Africa

Central and South America

Argentina

Bolivia

Brazil

Chile

Colombia

Ecuador

Falkland Islands

French Guiana

Guyana

Paraguay

Peru

Surinam

Uruguay

Venezuela

Belize

Costa Rica

El Salvador

Guatamala

Honduras

Mexico

Nicaragua

Panama

Antigua and Barbuda

Bahamas

Barbados

Cayman Islands

Cuba

Grenada

Guadeloupe

Jamaica

Netherlands Antilles

Puerto Rico

St Kitts-Nevis

St Lucia

St Vincent and the Grenadines

Trinidad and Tobago

Turks and Caicos Islands

Eastern Europe, Russia,

Central Asian and Baltic States

Bulgaria

Czechoslovakia

Hungary

Poland

Romania

Armenia

Azerbaijan

Belarus (formerly Byelorussia)

Estonia

Georgia

Kazakhstan

Kyrgyzstan (formerly Kirghizia)

Latvia

Lithuania

Moldova (formerly Moldavia)

Russian Federation

Ukraine

Uzbekistan

\section{Melanesia, Micronesia and Polynesia}

New Caledonia

Papua New Guinea

Solomon Islands

Vanuatu

Guam

Kiribati

Cook Islands

Fiji

French Polynesia (including

Tahiti)

Niue

American Samoa

Western Samoa

Tokelau

Tonga

Tuvalu

Wallis and Fortuna

\section{Middle East and Africa}

Bahrain

Gaza Strip

Iran

Iraq

Israel

Jordan

Kuwait

Lebanon

Qatar

Saudi Arabia

Syria

Turkey

United Arab Emirates

West Bank

Yemen

Algeria

Egypt

Libya

Mauritania

Morocco

Sudan

Tunisia

Cameroon

Central African Republic

Congo

Cote d'Ivoire

Gambia

Ghana

Guinea-Bissau

Liberia

Mali

Nigeria

Senegal

Sierra Leone

Zaire

Angola

Botswana

Djibouti

Ethiopia

Kenya

Malawi

Mauritius

Mozambique

Namibia

Reunion

Rwanda

Seychelles

Somalia

Swaziland

Tanzania

Uganda

Zambia

Zimbabwe
North East Asia

China (excluding Taiwan)

Hong Kong

Japan

North Korea

South Korea

Macau

Mongolia

Taiwan

\section{South East Asia}

Brunei

Cambodia

Indonesia

Laos

Malaysia

Burma (Myanmar)

Philippines

Singapore

Thailand

Vietnam

\section{Southern Asia}

Afganistan

Bangladesh

Bhutan

India

Maldives

Nepal

PakistanSri Lanka

\section{Southern Europe}

Albania

Andorra

Cyprus

Gibraltar

Greece

Italy

Malta

Portugal

Spain

Former Yugoslavia

(not otherwise defined)

Croatia

Slovenia

Western and Northern Europe

Austria

Belgium

France

Germany (United)

Luxembourg

Netherlands

Switzerland

Denmark

Faeroe Islands

Finland

Iceland

Norway

Sweden 
APPENDIX 4

NSW MIDWIVES DATA COLLECTION FORM

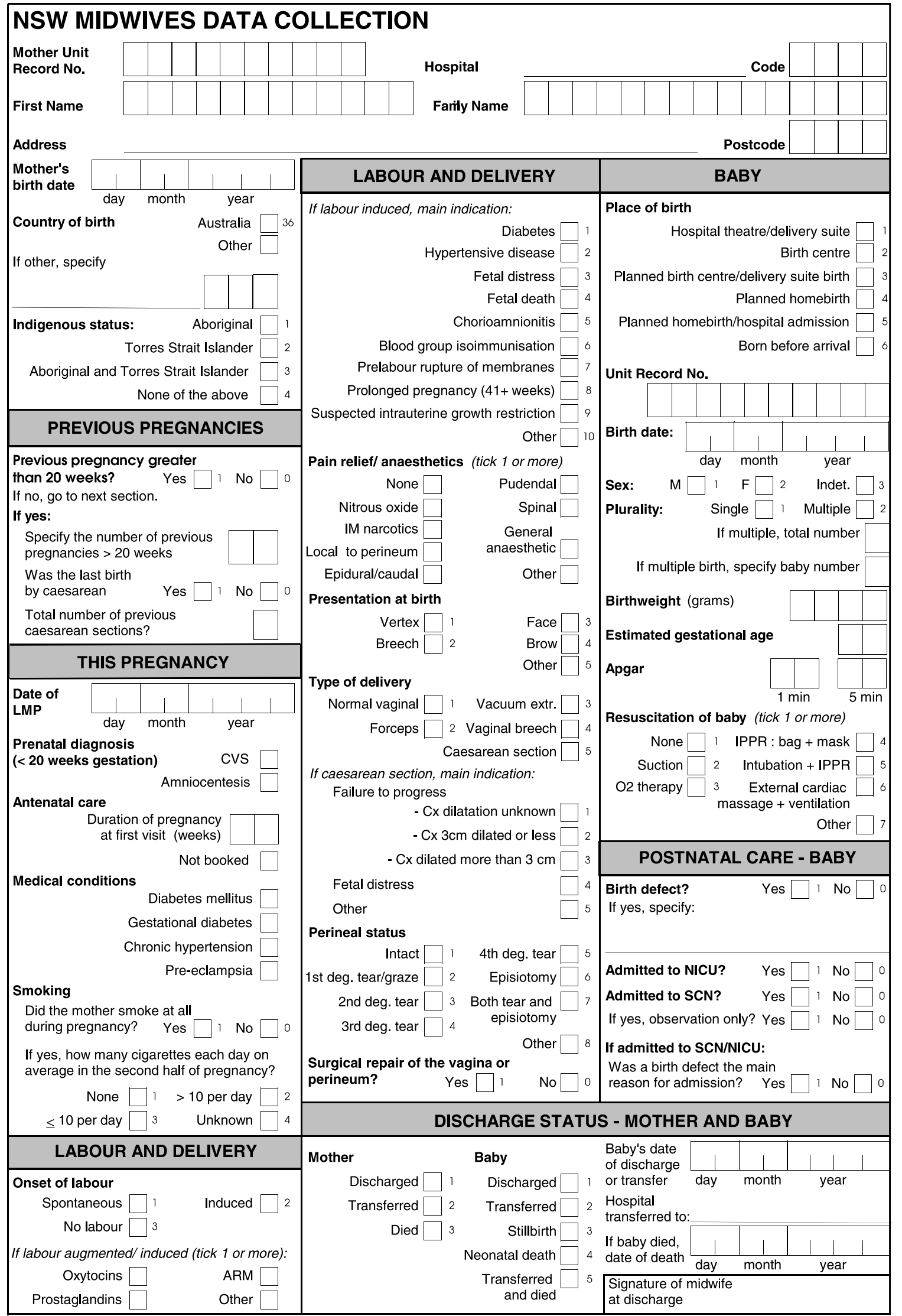

Health Department Copy

Please complete and forward to: NSW Midwives Data Collection

Patient Data Management Unit, Level 6

Locked Bag 961, North Sydney, NSW 2059 